\title{
Efeito do extrato de Passiflora Edulis na cicatrização da parede abdominal de ratos: estudo morfológico e tensiométrico ${ }^{1}$
}

\author{
Passiflora Edulis extract and the healing of abdominal wall of rats: morphological and \\ tensiometric study
}

\begin{abstract}
Cálide Soares Gomes², Antonio Carlos L. Campos ${ }^{3}$, Orlando Jorge Martins Torres², Paulo Roberto Leitão de Vasconcelos $^{3}$, Ana Tereza Ramos Moreira ${ }^{3}$, Sérgio Bernardo Tenório ${ }^{3}$, Elizabeth Milla Tâmbara ${ }^{3}$, Kenji Sakata ${ }^{5}$, Hugo Moraes Júnior ${ }^{4}$, André Luiz S. Ferrer ${ }^{4}$
\end{abstract}

1. Trabalho realizado no laboratório de Pesquisas do Centro de Ciências Biológicas e da Saúde da Universidade Federal do Maranhão

2. Professor do Departamento de Cirurgia da Universidade Federal do Maranhão

3. Professor Doutor em Cirurgia

4. Aluno de Pós-Graduação - Mestrado

5. Aluno de Pós-Graduação - Doutorado

\section{RESUMO}

Introdução: O emprego de plantas no tratamento de diversas moléstias é costumeiro no Brasil. O maracujá (Passiflora edulis) é muito empregado em diversas afecções, freqüentemente sem comprovação científica. Foram descritas propriedades antiinflamatórias do extrato de Passiflora edulis, semelhantes às dos antiinflamatórios não esteroidais (AINE's). Objetivo: Avaliar morfológica e tensiometricamente o efeito do extrato hidroalcoólico de Passiflora edulis no processo de cicatrização de laparotomias medianas em ratos. Métodos: Quarenta ratos Wistar, divididos em dois grupos de 20, foram submetidos à incisão e sutura da linha alba. Em 20 deles, grupo controle (GC), administrou-se solução salina na cavidade peritonial. Nos outros 20, grupo Passiflora (GP), administrou-se extrato de Passiflora edulis na cavidade peritonial, em dose única, isovolumétrica. O extrato foi obtido por extração alcoólica das folhas secas do maracujá. Os grupos controle e Passiflora foram subdivididos em dois, conforme o dia da morte: grupo controle três dias (C3), grupo controle sete dias (C7), grupo Passiflora três dias (P3) e grupo Passiflora sete dias (P7). Após a morte dos ratos, retirou-se espécimes da parede abdominal englobando a área operada. Os espécimes foram submetidos à avaliação macroscópica, histológica e tensiométrica. Resultados: Na avaliação macroscópica, não houve diferença significante entre GC e GP. Na histológica, comparando-se C3 vs. P3, a variável inflamação aguda foi mais intensa no grupo C3, enquanto a colagenização e a neoformação capilar apresentaram diferenças significantes (0,001 e 0,001, respectivamente), em favor de P3. Entre C7 vs. P7, as variáveis inflamação aguda, inflamação crônica e neoformação capilar também apresentaram diferenças significantes $(\mathrm{p}=0,002$; 0,006 e 0,001, respectivamente). Na avaliação tensiométrica, a carga máxima de ruptura (Cmáx) do sétimo dia foi maior no grupo Passiflora em relação ao grupo controle (6,91 $\pm 1,36$ vs. 5,05 $\pm 1,63, p=0,013)$. A deformação máxima de ruptura

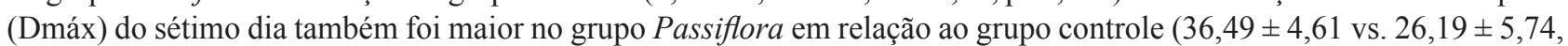
$\mathrm{p}=0,001)$. Conclusão: O extrato de Passiflora edulis melhora a cicatrização de laparotomias medianas em ratos, nos aspectos histológico e tensiométrico.

Descritores: Passiflora edulis. Fitoterapia. Cicatrização de Feridas. Ratos.

\section{ABSTRACT}

Introduction: The Brazilian popular habit of using plants to treat several health conditions is ancient. Passion fruit (Passiflora edulis) is widely used to treat, usually in an empiric basis, a variety of medical conditions. Anti-inflammatory activity of Passiflora edulis extract, similar to non-steroidal anti-inflammatory drugs (NSAID`s), has been described. Purpose: To evaluate the effect of Passiflora edulis extract hidroalcoolical on the healing of midline abdominal incisions in rats by morphological and tensiometric methods. Methods: Forty male Wistar rats were randomly allocated into two groups to either receive Passiflora edulis extract (study group, P) or saline (control group, C) intraperitoneally, in a single isovolumetric dose, after a standardized ventral midline laparotomy had been performed. The twenty rats of the control group as well as those twenty of the study group were divided into subgroups according to the time of sacrifice, either the 3rd PO day (P3, C3) or the 7th PO day (P7, C7). On day three and on day seven after surgery, the rats were sacrificed and the wound area was excised by a standardized protocol. The healing process of the specimens was evaluated macroscopically and histologically. The tensile strength was evaluated by a constant speed computerized tensiometer to determine the breaking strength and the deformation of the healing incision. Results: The macroscopic examination did not show significant differences between study and control groups. Histologically, the C3 vs. P3 comparison showed the following differences: for the variables acute inflammation ( $p=0.045$ in favor of C3), colagenization and capillary neoformation: $p=0.001$ e 0.001 , respectively in 
favor of P3. Similarly, the C7 vs. P7 comparison showed the following differences for the variables acute inflammation $(p=0.002$ in favor of 7 ), chronic inflammation and capillary neoformation: $p=0.006$ e 0.001 , respectively in favor of $\mathrm{P} 7$. Tensiometrically, maximal breaking strength (Cmáx) on day seven of the study group was higher when compared to control group, (6.91 \pm 1.36 vs. $5.05 \pm 1.63, \mathrm{p}=0.013)$. Maximal deformation strength (Dmáx) on day seven of the study group was higher when compared to control group (36.49 \pm 4.61 vs. $26.19 \pm 5.74, \mathrm{p}=0.001)$. Conclusion: Passiflora edulis extract enhances the healing of midline abdominal incisions in rats, especially the histological and tensiometric aspects.

Key words: Passiflora edulis. Phytotherapy. Wound Healing. Rats.

\section{Introdução}

O emprego de plantas no tratamento de diversas moléstias é prática milenar. Embora sem comprovação científica, ela persiste até os dias atuais, quando, inclusive, vários extratos de plantas são industrializados e comercializados, além dos artesanalmente empregados pela população. Apesar dessa transferência de conhecimento entre gerações, a maior parte das informações é fundamentada em experiências empíricas. Sua utilização, no entanto, vem sendo gradativamente regulamentada nas últimas décadas a partir de experimentos científicos que atestam a sua real eficácia e, sobretudo, determinam os principais efeitos colaterais.

O maracujá (Passiflora edulis) é planta muito comum no Brasil. Seus frutos são saborosos e muito usados como alimento e remédio pela população. Entre as substâncias químicas descritas na Passiflora edulis encontram-se os alcalóides (harmana, harmina, harmalina e harmol), os flavonóides e os carotenóides ${ }^{1,2,3}$.

Foram descritas propriedades sedativas, hipnóticas, analgésicas, antipiréticas, antiinflamatórias e parassimpaticolíticas em estudos com a Passiflora edulis e outras passifloráceas $^{4,5,6,7}$.

A cicatrização é um processo complexo e dinâmico de restauração de estruturas celulares e camadas teciduais. Divide-se nas fases inflamatória, proliferativa e de remodelação. Dentro destas três fases existe complexa e coordenada série de eventos que incluem quimiotaxia, fagocitose, neoformação, degradação e remodelação do colágeno. Além disso, angiogênese, epitelização e produção de novos glicosaminoglicanos e proteoglicanos são vitais para o meio onde ocorre a cicatrização. Estes processos biológicos resultam, finalmente, na substituição dos tecidos lesados por tecido cicatricial $^{8}$. Foi sugerida a presença de compostos químicos no extrato de Passiflora edulis, em particular, substâncias com propriedades farmacológicas semelhantes às dos antiinflamatórios não-esteroidais ${ }^{6,7}$.

$\mathrm{O}$ efeito de antiinflamatórios não-esteroidais na cicatrização da parede abdominal de ratos já foi objeto de estudos experimentais com resultados conflitantes ${ }^{9,10,11}$.

Como foi sugerida ação antiinflamatória não-esteroidal do extrato hidroalcoólico de Passiflora edulis em ratos ${ }^{7}$, o objetivo deste estudo é avaliar o efeito do seu extrato na cicatrização da parede abdominal de ratos mediante análise morfológica e tensiométrica.

\section{Métodos}

Este estudo foi realizado no Laboratório de Pesquisa do Departamento de Fisiologia e Farmacologia da Universidade Federal do Maranhão (UFMA), São Luiz, MA, Brasil, onde os animais foram mantidos durante todo o período experimental e onde também coletaram-se os dados para avaliação clínica, morfológica e tensiométrica. O trabalho foi conduzido respeitando-se a legislação brasileira para o uso de animais de experimentação - Lei Federal n ${ }^{\circ} 6.638$ (1979) e as normas do Colégio Brasileiro de Experimentação Animal (COBEA). O protocolo foi aprovado pelo Comitê de Ética em Pesquisa da UFMA.

\section{Amostra}

Foram utilizados 40 ratos (Rattus norvegicus albinus, Rodentia mammalia), da linhagem Wistar, machos, entre 30 e 45 dias de idade, pesando entre 80 e 110 gramas, saudáveis, oriundos do Biotério da Universidade Federal do Maranhão. A amostra foi aleatoriamente distribuída em dois grupos de 20 ratos: grupo Passiflora (GP) e grupo controle (GC), segundo o tratamento recebido: extrato de Passiflora edulis ou solução salina. Cada um destes dois grupos foi subdividido em subgrupos de 10 ratos, segundo o dia da morte: subgrupo Passiflora 3 dias (P3), subgrupo Passiflora 7 dias (P7), subgrupo controle 3 dias (C3) e subgrupo controle 7 dias (C7), conforme a Figura 1.

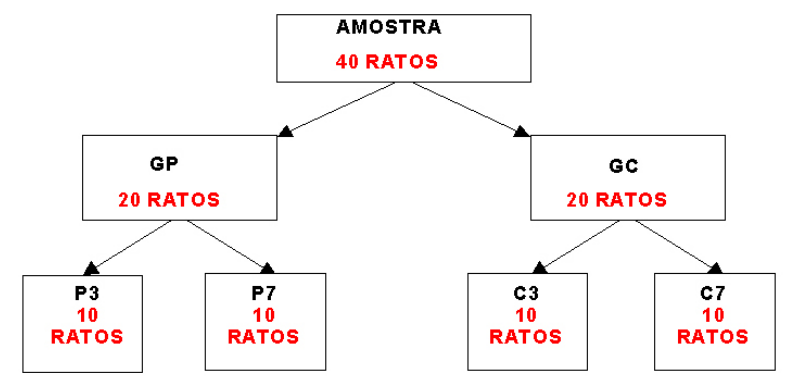

FIGURA 1 - Distribuição da amostra

\section{Preparo do extrato de Passiflora edulis}

Foram colhidas cerca de oito quilogramas de folhas íntegras de maracujá (Passiflora edulis), acondicionadas em sacos plásticos e transportadas até o laboratório de Farmacologia da UFMA. O material foi identificado e catalogado no Herbário Ático Seabra, da UFMA, sob o número 1155. As folhas foram colocadas para secar à temperatura ambiente por duas semanas. Em seguida, colocadas em estufa 
de secagem para retirada da umidade sob temperatura de 45 a $50^{\circ} \mathrm{C}$, por 24 horas. Posteriormente, as folhas foram submetidas ao processo de moagem em moinho elétrico (Tecnal TE-650 ${ }^{\circledR}$, São Paulo-SP), no Laboratório do Pavilhão Tecnológico da UFMA. Obteve-se $2000 \mathrm{~g}$ de um pó de cor e odor característicos. Este pó foi colocado em recipiente adequado e em seguida adicionou-se $6000 \mathrm{ml}$ de solução hidroalcoólica a 70\%. Esta mistura foi manualmente agitada por cinco minutos a cada duas horas, durante 12 horas, após o que foi filtrada em funil de vidro simples e algodão por três vezes consecutivas, obtendo-se, no final dessa etapa, $5500 \mathrm{ml}$ de extrato bruto.

A concentração do extrato bruto foi calculada em $\mathrm{g} / \mathrm{ml}$. O extrato bruto foi concentrado em evaporador rotativo (Fisaton $802^{\circledR}$ São Paulo-SP) a uma temperatura de 60 a $65^{\circ} \mathrm{C}$ para eliminação total do solvente. $\mathrm{O}$ material obtido depois da concentração se apresentou em forma de pasta. Deste, foram retiradas amostras para testes farmacológicos no Laboratório de Bioquímica do Centro de Ciências Biológicas e da Saúde da UFMA, nas concentrações desejadas.

Para a determinação da concentração $(\mathrm{g} / \mathrm{mI})$ desse extrato, foi calculado o seu peso seco. Em três beckeres de peso determinado, adicionou-se um mililitro do extrato em cada um e em seguida, evaporou-se o solvente em corrente de ar quente (secador de cabelos comercial). Os pesos das amostras foram posteriormente determinados em balança analítica digital (AL-200 ${ }^{\circledR}$, São Paulo-SP) no Laboratório de Produtos Naturais da UFMA. A determinação do peso foi realizada por mais três vezes até estabilização dos valores. A média aritmética dos pesos dos beckeres correspondeu ao peso seco do extrato.

O extrato obtido foi enviado para análise no Departamento de Tecnologia Química do Laboratório de Controle de Qualidade de Alimentos da UFMA, que assim determinou sua constituição: umidade: 58,63\%; açúcares totais: 19,20\%; proteínas: 9,98\% gorduras: $6,54 \%$ e cinzas: $5,65 \%$. O pH do extrato foi 5,10. Para os testes farmacológicos intraperitoneais foram retirados $20 \mathrm{~g}$ do extrato bruto hidroalcoólico e diluiu-se em $100 \mathrm{ml}$ de solução salina, que resultou em concentração de $200 \mathrm{mg} / \mathrm{ml}$. Esta solução foi mantida refrigerada a $4^{\circ} \mathrm{C}$.

\section{Pré-operatório e anestesia}

Os ratos foram mantidos no biotério, cinco animais por gaiola, em condições de iluminação, temperatura e umidade ambientais, com livre acesso a ração própria para ratos (Purina $^{\circledR}$, Paulínia-SP) e água potável ad libitum durante sete dias para adaptação. Foram mantidos em jejum de oito horas antes da anestesia.

Os animais foram anestesiados com éter sulfúrico, por inalação em câmara fechada até a obtenção do plano anestésico, comprovado pela ausência de movimentos voluntários e sem reação ao manuseio. O plano anestésico foi mantido por inalação de éter sulfúrico, sob máscara em sistema artesanal aberto.

Os ratos foram pesados e identificados por tatuagem com ácido pícrico. Em seguida, foram fixados em decúbito dorsal horizontal sobre prancha cirúrgica de madeira, medindo 20 por $30 \mathrm{~cm}$, e imobilizados por meio de tensores elásticos, presos aos quatro membros e ancorados aos quatro cantos da prancha cirúrgica.

\section{Ato operatório}

Com o animal anestesiado, realizou-se epilação por tração manual dos pelos da região ventral do abdome em área de 4,0 por 3,0 cm, anti-sepsia da região abdominal e torácica por aplicação com gaze embebida em polivinilpirrolidonaiodo (Povidine Tópico ${ }^{\circledR}$, Rio de Janeiro-RJ) e colocação de campo fenestrado estéril sobre o animal, expondo a região abdominal. Realizou-se incisão longitudinal mediana de três cm de extensão a partir do apêndice xifóide, com lâmina de bisturi número 15 e tesoura reta, interessando pele, tela subcutânea, linha alba (plano músculo-aponeurótico) e peritônio. Após abertura da cavidade abdominal, injetou-se, com seringa de $1 \mathrm{ml}$, diretamente na cavidade peritonial, extrato de Passiflora edulis ou solução salina, segundo o grupo ao qual pertencia o animal, GP ou GC. Os ratos do GP receberam extrato de Passiflora edulis na concentração de $200 \mathrm{mg} / \mathrm{kg} /$ peso em dose única, enquanto os ratos do GC receberam solução salina a $0,9 \%$, em dose única, isovolumétrica. Em seguida, procedeu-se à síntese da parede abdominal do seguinte modo: no plano músculo-aponeurótico e peritoneal utilizou-se fio de polipropileno monofilamentado azul 5-0 (Prolene ${ }^{\circledR}$, Ethicon, New Jersey, EUA), montado em agulha atraumática, curva (1/2 círculo), cilíndrica, $1,5 \mathrm{~cm}$ de comprimento. A sutura consistiu de seis pontos simples, separados, a $0,5 \mathrm{~cm}$ das bordas da ferida, espaçamento de $0,5 \mathrm{~cm}$ entre os pontos. Cada ponto foi atado com quatro nós. A pele foi suturada do mesmo modo, com seis pontos contínuos, com o mesmo fio (Figura 2).

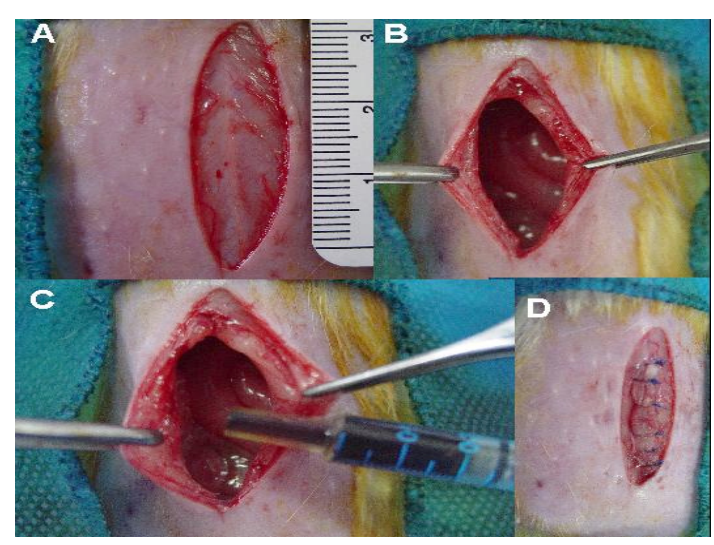

FIGURA 2 - Detalhes da operação. Nota: A) incisão da linha alba. B) linha alba aberta. C) injeção do extrato de Passiflora edulis. D) síntese da linha alba

A ferida foi limpa com solução de cloreto de sódio a $0,9 \%$ e, em seguida, com gaze embebida em polivinilpirrolidona-iodo (Povidine Tópico ${ }^{\circledR}$ ).

\section{Pós-operatório}

Ao final do ato operatório, os ratos foram colocados nas 
suas respectivas gaiolas, em condições semelhantes às do período pré-operatório. Ração e água estavam disponíveis, ad libitum, no pós-operatório imediato. As gaiolas foram identificadas com o dia da operação e o tipo de tratamento recebido (solução salina ou extrato de Passiflora edulis). Os animais foram examinados diariamente, anotando-se as condições da ferida e as possíveis intercorrências.

Os animais do GC foram divididos em dois subgrupos de 10 ratos, $\mathrm{C} 3$ e $\mathrm{C} 7$, com morte prevista para o terceiro e sétimo dia de pós-operatório, respectivamente. Os do GP foram divididos em dois subgrupos de 10 ratos, P3 e P7, com morte prevista para o terceiro e sétimo dia de pós-operatório, respectivamente.

\section{Avaliação macroscópica e obtenção dos espécimes}

Após a morte por inalação contínua de éter os animais foram pesados e fixados à prancha cirúrgica, do mesmo modo que no ato operatório. Obteve-se o espécime cirúrgico mediante incisão retangular com dimensões de 4,5 cm crânio-caudal e $3,5 \mathrm{~cm}$ látero-lateral, compreendendo a incisão operatória e tecidos circunjacentes, interessando toda a espessura da parede abdominal (Figura 3).

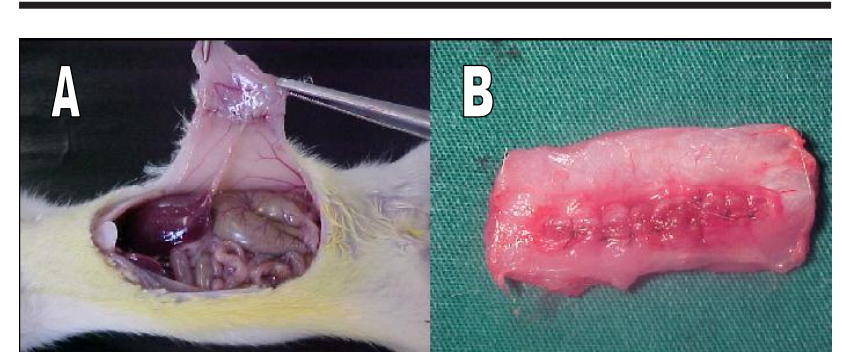

FIGURA 3 - Detalhes da obtenção do espécime (corpo de prova). Nota: A) espécime sendo dissecado. B) espécime já retirado, sobre campo cirúrgico

Pela abertura realizada na parede abdominal, promoveuse inventário da cavidade peritoneal, anotando-se a presença de abscessos e deiscências de sutura.

As amostras cirúrgicas (espécimes) retiradas foram lavadas em água corrente e os fios cirúrgicos foram removidos utilizando-se pinça anatômica e lâmina de bisturi. Das extremidades superiores dos espécimes retirou-se faixa horizontal de um centímetro para estudo histológico, que foi colocada em frasco apropriado contendo formol a $10 \%$. O restante do espécime foi acondicionado em frasco apropriado contendo solução salina a $0,9 \%$ para estudo tensiométrico. Os frascos foram marcados com o grupo, subgrupo e identificação do animal.

\section{Avaliação microscópica}

Os fragmentos dos espécimes cirúrgicos destinados à avaliação histológica foram fixados em formol a $10 \%$ e encaminhados para o Laboratório de Histologia da UFMA, e corados pela técnica de Hematoxilina-Eosina (HE). Examinou-se a área de sutura considerando-se os processos de reparação tecidual e inflamatório da linha de sutura. Estudaram-se oito campos por lâmina, com as objetivas de 4, 10 e 40 vezes e ocular de 10 vezes. Os parâmetros ou variáveis histológicas consideradas foram avaliadas segundo a classificação proposta por COTRAN et al. ${ }^{12}$ : inflamação aguda, inflamação crônica inespecífica, necrose isquêmica, reação gigantocelular do tipo corpo estranho, proliferação fibroblástica, fibrose (colagenização), reepitelização, coaptação das bordas da sutura, extensão do infiltrado e neoformação capilar.

\section{Avaliação tensiométrica}

As amostras cirúrgicas (espécimes) destinadas ao estudo tensiométrico foram encaminhadas para o Laboratório de Mecânica e Materiais do Centro Federal de Educação Tecnológica do Maranhão (CEFET-MA), onde foi realizado o experimento.

Para o ensaio de tração da parede anterior do abdome dos ratos foi utilizada máquina universal de ensaios, computadorizada (modelo TT 2420, TIRA Maschinenbau, Alemanha) do Laboratório de Ensaios Mecânicos do CEFET-MA. Essa máquina é equipada com célula de carga com capacidade máxima de $20 \mathrm{kN}$ e possui precisão melhor que $0,5 \%$. As deformações foram medidas mediante deslocamento do carro superior do equipamento.

Antes do ensaio propriamente dito, todas as peças receberam pré-carga de $2 \mathrm{~N}$, com tempo de acomodação de 60 segundos. A velocidade estabelecida para todos os ensaios foi de cinco milímetros por minuto e as medidas foram realizadas a cada $0,5 \mathrm{~mm}$. Após a fixação nos acessórios da máquina universal de ensaios, as amostras foram submetidas a tração axial (Figura 4).

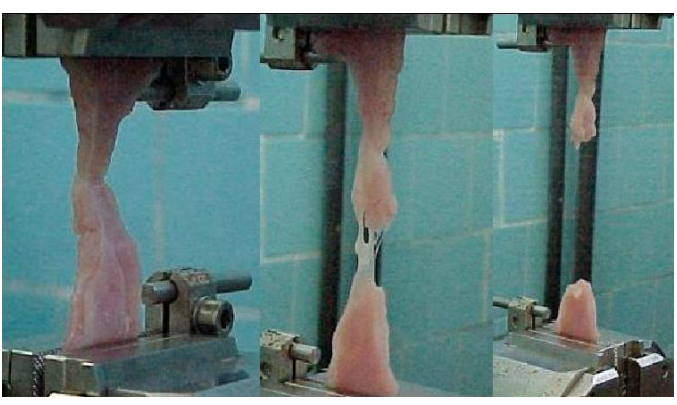

FIGURA 4 - Seqüência de rotura de um corpo de prova, submetido a ensaio tensiométrico

Os ensaios foram realizados até ser atingido decréscimo de $50 \%$ do valor da carga máxima, registrando-se as cargas correspondentes a cada deformação medida. Os parâmetros estudados foram a carga máxima de ruptura (Cmáx), medida em Newtons (N) e a deformação máxima de ruptura (Dmáx), medida em milímetros ( $\mathrm{mm})$.

\section{Análise estatística}

Os dados obtidos das avaliações macro e microscópica e dos estudos tensiométricos foram submetidos aos testes de 
estatística descritiva para o tratamento básico dos dados e testes estatísticos específicos, quando aplicáveis (CALLEGARI-JACQUES, 2003). As variáveis foram comparadas entre os subgrupos, ou seja, C3 vs. P3, C7 vs. P7 e dentro do mesmo subgrupo, ou seja, C3 vs. C7 e P3 vs. P7.

As variáveis numéricas deformação máxima de ruptura (DMáx) e carga máxima de ruptura (CMáx) foram avaliadas pelo teste $t$ de Student e por uma ANOVA com dois fatores (Grupo - GC e GP) e (Tempo - 3 e 7 dias) e a interação entre eles. As variáveis ordinais (classificatórias) das avaliações macro e microscópica foram analisadas pelo teste não-paramétrico de Mann-Whitney.

Os dados foram analisados utilizando-se o programa Statistica for Windows 5.1 (StatSoft, Inc., Tulsa, EUA).

Os resultados foram expressos em média \pm desvio-padrão (DP). Em todos os testes fixou-se em 0,05 ou $5 \%, \alpha$ (alfa) < 0,05 o nível para rejeição da hipótese de nulidade.

\section{Resultados}

Nenhum rato evoluiu com fístulas, deiscências ou abscessos da ferida cirúrgica da parede abdominal.

\section{Avaliação microscópica}

Aos três dias de pós-operatório, o subgrupo Passiflora diferiu estatisticamente do subgrupo controle em três das dez variáveis histológicas comparadas: inflamação aguda (em favor de C3), colagenização e neoformação capilar (em favor de P3).

A Tabela 1 apresenta os resultados da comparação das variáveis histológicas do terceiro dia entre os subgrupos controle e Passiflora.

TABELA 1 - Análise das variáveis histológicas do $3^{\circ}$ dia (análise intergrupo, c3 vs p3)

\begin{tabular}{lccc}
\hline Variável histológica & C3 & P3 & p \\
\hline Inflamação aguda & 131,5 & 78,5 & $0,045^{*}$ \\
Inflamação crônica & 95 & 115 & 0,450 \\
Necrose isquêmica & 119 & 91 & 0,290 \\
Reação gigantocelular & 113 & 97 & 0,545 \\
Proliferação fibroblástica & 83 & 127 & 0,096 \\
Colagenização & 59 & 151 & $0,001^{*}$ \\
Reepitelização & 105 & 105 & 1,000 \\
Coaptação das bordas & 100 & 110 & 0,705 \\
Extensão do infiltrado & 105 & 105 & 1,000 \\
Neoformação capilar & 55 & 155 & $0,001^{*}$ \\
\hline
\end{tabular}

Notas: Os valores de $\mathrm{p}^{*}$ indicam diferenças estatisticamente significantes.

Utilizou-se o teste não-paramétrico de Mann-Whitney.

Legenda: $\mathrm{p}=$ nível de significância estatística.

Aos sete dias de pós-operatório os resultados foram similares. O subgrupo Passiflora apresentou diferença estatisticamente significante do subgrupo controle em três das dez variáveis histológicas comparadas: inflamação aguda (em favor de C7), inflamação crônica e neoformação capilar (em favor de P7).

A Tabela 2 apresenta o resultado da comparação das variáveis histológicas do sétimo dia entre os subgrupos controle e Passiflora.

TABELA 2 - Análise das variáveis histológicas do $7^{\circ}$ dia (análise intergrupo, C7 vs P7)

\begin{tabular}{lccc}
\hline Variável histológica & C7 & P7 & p \\
\hline Inflamação aguda & 130 & 41 & $0,002^{*}$ \\
Inflamação crônica & 64 & 107 & $0,006^{*}$ \\
Necrose isquêmica & 80 & 91 & 0,183 \\
Reação gigantocelular & 85,5 & 85,5 & 0,399 \\
Proliferação fibroblástica & 106 & 65 & 0,328 \\
Colagenização & 94 & 77 & 0,929 \\
Reepitelização & 107 & 64 & 0,286 \\
Coaptação das bordas & 115 & 56 & 0,076 \\
Extensão do infiltrado & 95 & 766 & 1,000 \\
Neoformação capilar & 59 & 112 & $0,001^{*}$ \\
\hline
\end{tabular}

Notas: Os valores de $\mathrm{p}^{*}$ indicam diferenças estatisticamente significantes. Utilizou-se o teste não-paramétrico de Mann-Whitney.

Legenda: $\mathrm{p}=$ nível de significância estatística.

\section{Carga máxima de ruptura}

Todas as amostras romperam-se ao nível da sutura. Não houve diferença significativa na carga máxima de ruptura (Cmáx) Cmáx entre C3 e P3 (4,83 $\pm 1,76$ N vs. 5,56 \pm 2,28 $\mathrm{N})$. Entretanto, comparando-se a carga máxima de ruptura Cmáx entre C7 e P7 $(5,05 \pm 1,63 \mathrm{~N}$ vs. $6,91 \pm 1,36 \mathrm{~N})$, a diferença foi estatisticamente significante em favor de P7, $\mathrm{p}=0,013$, como ilustra a Figura 5 e Tabela 3 .

\section{Deformação máxima de ruptura}

Comparando-se a Dmáx entre C3 e P3 (24,99 \5,20 mm vs. $25,92 \pm 2,69 \mathrm{~mm}$ ), a diferença não foi estatisticamente significante, $p=0,623$. Em contraste, comparando-se a Dmáx entre C7 e P7 $(26,19 \pm 5,74 \mathrm{~mm}$ vs. $36,49 \pm 4,61 \mathrm{~mm})$, ela foi estatisticamente significante em favor de P7, $\mathrm{p}=0,001$.

TABELA 3 - Cmáx, EM N. Média \pm DP, comparação intergrupo e intragrupo

\begin{tabular}{lccc}
\hline & 3 Dias & 7 Dias & p (evolução) \\
\hline Controle & $4,83 \pm 1,76$ & $5,05 \pm 1,63$ & 0,779 \\
Passiflora & $5,56 \pm 2,28$ & $6,91 \pm 1,36$ & 0,126 \\
\hline
\end{tabular}

Notas: $\mathrm{O}$ valor de $\mathrm{p}$ em negrito indica diferença estatisticamente significante. Utilizou-se o teste $t$ de Student.

Legenda: $\mathrm{p}$ = nível de significância estatística

A Tabela 4 ilustra não ter havido diferença estatisticamente significante entre os valores da Dmáx quando se comparou C3 com P3 ou C3 com C7. Porém, a diferença foi estatisticamente significante quando se comparou $\mathrm{C} 7$ com P7, em favor de P7 e quando se comparou P3 com P7, em favor de $\mathrm{P} 7$. 


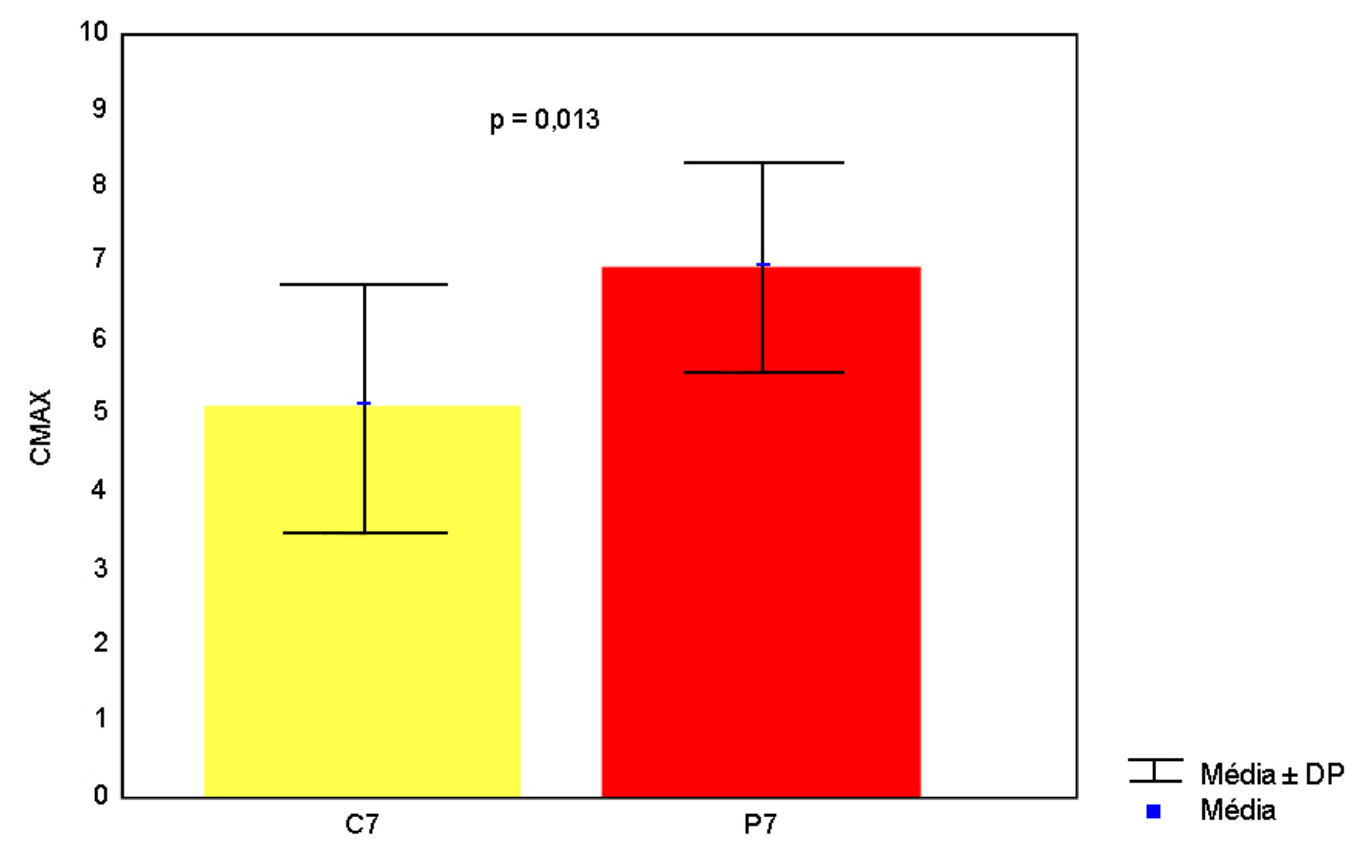

FIGURA 5 - Média \pm DP DA Cmáx Entre C7 E P7

TABELA 4 - Dmáx, EM mm. Média \pm DP, comparação intergrupo e intragrupo

\begin{tabular}{lccc}
\hline & 3 Dias & 7 Dias & $\begin{array}{c}\mathbf{p} \\
\text { (evolução) }\end{array}$ \\
\hline Controle & $4,83 \pm 1,76$ & $5,05 \pm 1,63$ & 0,779 \\
Passiflora & $5,56 \pm 2,28$ & $6,91 \pm 1,36$ & 0,126 \\
$p$ (intergrupo) & 0,623 & $0,001 *$ & \\
\hline
\end{tabular}

Notas: O valores de $\mathrm{p}^{*}$ indicam diferenças estatisticamente significantes. Utilizou-se o teste $t$ de Student.

Legenda: $\mathrm{p}$ = nível de significância estatística

\section{Discussão}

O extrato de Passiflora edulis foi escolhido como droga de teste neste estudo por ter demonstrado atividade antimicrobiana, antiinflamatória e analgésica em estudos farmacológicos anteriores. De fato, Nicolls et al. ${ }^{13}$, Silva ${ }^{6}$ e Silva $^{7}$ utilizaram extrato de Passiflora edulis como antinflamatório, antimicrobiano ou hipnótico em diversos estudos experimentais.

No presente estudo, o extrato de Passiflora edulis mostrou-se de fácil obtenção, conservação e administração. A dose escolhida, $200 \mathrm{mg} / \mathrm{kg}$, baseou-se em valor situado entre as menores $(100 \mathrm{mg} / \mathrm{kg})$ e as maiores $(1000 \mathrm{mg} / \mathrm{kg})$ rotineiramente empregadas em estudos farmacológicos com a Passiflora edulis ${ }^{6,7}$, assim como, nos mesmos estudos, a via de administração mais empregada por sua facilidade e eficiência tem sido a intraperitonial.

Pela rapidez e simplicidade de administração, também empregou-se a via intraperitonial. Escolheu-se a dose de $200 \mathrm{mg} / \mathrm{kg}$, valor menor que o da dose máxima habitualmente empregada nos estudos com Passiflora edulis. A escolha de doses maiores acarretaria maiores volumes a serem injetados intraperitonialmente, o que poderia alterar os resultados. Optou-se por administrar a dose escolhida apenas uma vez, para evitar manipulações desnecessárias dos ratos. A existência de diferenças significantes entre os grupos estudados comprova a eficiência da administração do extrato de Passiflora edulis em dose única.

Escolheu-se a avaliação histológica do processo cicatricial por se tratar de método consagrado de estudo de cicatrização em cirurgias experimentais ${ }^{10,11}$. A coloração pela Hematoxilina-Eosina é simples e barata, adequada para quantificar e identificar os elementos celulares envolvidos nas fases inflamatória, proliferativa e de remodelamento do processo cicatricial. A reação inflamatória é importante para a cicatrização; é a fase inicial desta. Porém, constitui-se em fator nocivo se for intensa, pelo comprometimento da microcirculação local e da proliferação de fibroblastos.

A escolha de um segundo método de aferição do processo cicatricial, a tensiometria, revela-se bastante pertinente já que tem sido empregada em diversos estudos sobre cicatrização da parede abdominal, além de compensar certa subjetividade existente nos estudos sobre cicatrização em que apenas a avaliação histológica é empregada ${ }^{10,14}$.

O fato de nenhum dos animais ter evoluído com fístulas, deiscências ou abscessos da ferida cirúrgica da parede abdominal, pelo menos pressupõe pequena ou nula capacidade do extrato de Passiflora edulis em predispor ou induzir tais condições. Macroscopicamente, na cicatrização da parede abdominal de ratos, extrato de Passiflora edulis e solução salina são similares. É possível que a avaliação macroscópica não tenha sido, isoladamente, suficiente para demonstrar pequenas diferenças entre os grupos estudados.

As três únicas variáveis histológicas que apresentaram diferenças estatisticamente significantes entre C3 e P3, inflamação aguda em favor de C3 e colagenização e neoformação capilar em favor de P3, demonstram que, aos três dias de 
pós-operatório, o extrato de Passiflora edulis promove tanto a colagenização quanto a neoformação capilar, ao mesmo tempo em que diminui a inflamação aguda. $\mathrm{O}$ aumento tanto do colágeno quanto da neoformação capilar, contribuem para uma cicatriz mais forte. Inegavelmente, uma fase inflamatória aguda mais intensa e demorada compromete a complexa cadeia de eventos da cicatrização 12 .

Na comparação entre C7 e P7, as três únicas variáveis histológicas que apresentaram diferenças estatisticamente significantes, inflamação aguda em favor de C7 e inflamação crônica e neoformação capilar em favor de P7, demonstram que, aos sete dias de pós-operatório, o extrato de Passiflora edulis continua promovendo a neoformação capilar e diminuindo a inflamação aguda, ao mesmo tempo em que aumenta o índice de inflamação crônica. Novamente, persiste aqui favorecimento da cicatrização pela redução do índice de inflamação aguda e pelo aumento da neoformação capilar.

O uso do extrato de Passiflora edulis também associou-se a aumento da resistência à tração, tanto na carga quanto na deformação de ruptura.

Com relação à carga máxima de ruptura, quando se comparou $\mathrm{C} 3$ versus $\mathrm{P} 3$, a pequena diferença em favor de P3, embora sem significância estatística, mostra tendência de aumento da resistência à tração no subgrupo tratado com extrato de Passiflora edulis. Quando se comparou a carga máxima de ruptura entre C7 e P7, encontrou-se diferença estatisticamente significante em favor de P7, o que, confirmou a tendência de aumento da resistência à tração que se apresentou na comparação C3 versus P3. Depreende-se que o mecanismo da ação favorecedora do extrato de Passiflora edulis no que toca ao aumento da força de resistência à ruptura, manifesta-se mais claramente nas fases mais tardias do processo de cicatrização.

Similarmente, a deformação máxima de ruptura, quando se comparou C3 versus P3, a pequena diferença em favor de P3, embora sem significância estatística, novamente mostra tendência de aumento da deformação à tração no subgrupo tratado com extrato de Passiflora edulis. Pelo menos, em valores absolutos, a média de $\mathrm{P} 3$ superou a média de $\mathrm{C} 3$.

A deformação máxima de ruptura, comparada entre $\mathrm{C} 7$ e P7, revelou diferença estatisticamente significante em favor de P7. Aqui, novamente, a tendência que se apresentou na comparação C3 versus P3 é observada. Mais uma vez, infere-se que o extrato de Passiflora edulis exerça sua ação nas fases mais tardias do processo de cicatrização, tal como ocorreu com a carga máxima de ruptura.

Os mecanismos pelos quais o extrato de Passiflora edulis interfere favoravelmente no processo cicatricial ainda não são bem conhecidos. Sabe-se que as Passifloras apresentam efeito narcotizantes. As folhas de muitas delas fornecem tinturas e decoctos que têm ação semelhante à da morfina.

Nicolls et $\mathrm{al}^{13}$ estudou as atividades antibacterianas e antifúngicas do Passicol, substância encontrada nos tecidos vivos de espécies de Passiflora. Discos de sensibilidade impregnados com extrato de P. molissima e pedaços vivos do caule de P. coerulea foram colocados sobre placas de Petri contendo ágar ou ágar-sangue previamente semeados fungos e bactérias. Observaram que os halos de inibição do crescimento das colônias de microorganismos nas placas de
Petri superou os dos controles. Concluíram que o Passicol contido no extrato de Passiflora molissima inibe o crescimento de vários microorganismos in vitro.

Borreli $^{5}$ avaliou a atividade antiinflamatória da Passiflora incarnata, mediante estudo da ação do seu extrato etanólico em ratos. Empregou os testes do edema de pata provocado pela carragenina, pleurisia induzida pelo dextran e indução de granulomas por chumaços de algodão. $\mathrm{O}$ extrato etanólico de Passiflora incarnata foi administrado aos ratos por via oral. $\mathrm{O}$ edema de pata provocado pela carragenina foi reduzido pela administração de Passiflora incarnata. $\mathrm{Na}$ pleurisia induzida pelo dextran, a migração de leucócitos foi acentuadamente reduzida nos ratos tratados com Passiflora incarnata, claramente devido à importante supressão da migração de polimorfonucleares e mononucleares. No teste de indução de granulomas por chumaços de algodão, a Passiflora incarnata reduziu significativamente o peso dos granulomas apenas em altas doses. Concluíram que o extrato de Passiflora incarnata exibe atividade antiinflamatória.

Silva ${ }^{6,7}$ realizou estudos da Passiflora edulis para caracterizar sua ação farmacológica quanto às atividades depressora do sistema nervoso central, analgésica, antiinflamatória e antiespasmódica. Obteve o extrato das folhas secas de Passiflora edulis por extração etanólica. O autor concluiu que o extrato etanólico de Passiflora edulis possui compostos com atividade depressora do sistema nervoso central; possui princípio ativo com atividade analgésica e antiinflamatória semelhante à dos antiinflamatórios não-esteroidais e possui provável atividade anticolinérgica e inibitória do sistema digestório.

\section{Conclusão}

O extrato de Passiflora edulis favorece a cicatrização de laparotomias medianas em ratos, reduzindo a inflamação aguda e promovedo colagenização e neoformação capilar, além de aumentar a força de ruptura à tração e a deformação de ruptura à tração.

\section{Referências}

1. Lutomski J, Malek B, Stachowiak Z. Pharmacochemical investigations of the raw materials from Passiflora genus. 1. New method of chromatographic separation and fluorometric-planimetric determination of alkaloids and flavonoids in harman raw materials. Planta Med. 1974;26:311-7.

2. Lutomski J, Malek B. Pharmacochemical investigations on raw materials of genus Passiflora. 3. Phytochemical investigations on raw materials of Passiflora edulis forma flavicarpa. Planta Med. 1975;27:222-5.

3. Lutomski J, Malek B, Rybacka L. Pharmacochemical investigations of the raw materials from Passiflora genus. 2. The pharmacochemical estimation of juices from the fruits of Passiflora edulis and Passiflora edulis forma flavicarpa. Planta Med. 1975;27:112-21. 
4. Maluf E, Barros HMT, Frochtengarten R, Benti R, Leite JR. Assesment of the hypnotic/sedative effects and toxicity of Passiflora edulis aqueous extract in rodents and humans. Phytother Res. 1991;5:262-6.

5. Borreli F, Pinto L, Izzo AA, Mascolo N, Capaso F, Mercati V, Toja E, Autore G. Anti-inflammatory activity of Passiflora incarnata L in rats. Phytother Res. 1996;10:104-6.

6. Silva BTF. Estudos Farmacológicos da Passiflora edulis. [Monografia - Especialização]. São Luis (MA): Universidade Federal do Maranhão - Centro de Ciências da Saúde; 2000.

7. Silva BTF, Nunes SFLC, Freire SMF. Efeito antiinflamatório, analgésico e antipirético do extrato etanólico de folhas de Passiflora edulis var. flavicarpa (maracujáamarelo). Cad Pesq. 2001;12:28-37.

8. Mercandetti M, Cohen AJ. Wound Healing, Healing and Repair. Disponível em URL: $<$ http://www.emedicine. com/plastic/topic411>Acesso em 11 ago. 2003.

9. Tognini JRF, Goldenberg S, Simões MJ, Sauer L, Melo RL, Ortiz PLA. Efeito do diclofenaco de sódio na cicatrização da parede abdominal de ratos. Acta Cir Bras.1998;13:1-8.

10. Tognini JRF, Fagundes DJ, Novo NF, Juliano Y. Estudo biomecânico e morfológico da cicatrização da parede abdominal sob ação de meloxicam. Acta Cir Bras. 2000;15:10-5.

11. Minossi JG, Leite CV de S, Naresse LE, Rodrigues MAM, Angeleli AYO, Kobayasi S. Efeito do diclofenaco de sódio na cicatrização da parede abdominal de ratos. Estudo histopatológico, da força de ruptura e do colágeno tecidual. Acta Cir Bras. 2001;16;10-5.

12. Cotran RS, Kumar V, Collins T, Robbins S L. Robbins Pathologic Basis of Disease. Philadelphia: WB Saunders; 1999.
13. Nicolls JM, Birner J, Forsell P. Passicol, an antibacterial and antifungal agent produced by Passiflora plant species: qualitative and quantitative range of activity. Antimicrob Agents Chemoter. 1973;3:105-9.

14. Seid MH, McDaniel-Owens LM, Poole GV Jr., Meeks GR. A randomized trial of abdominal incision suture technique and wound strength in rats. Arch Surg. 1995; 130;394-7.

15. Cohen IK, Diegelmann RF, Crossland MC. Os cuidados com a ferida. In: Sabiston Jr., DC. Tratado de Cirurgia. Rio de Janeiro: Interamericana; 1986. p. 251-73.

16. Benet LZ, Kroetz DL, Sheiner LB. "Pharmacokinetics: The dynamics of drug absorption, distribution, and elimination". In: Molinoff PB, Ruddon RW, editores The pharmacological basis of therapeutics. McGraw-Hill; 1996.

17. Thornton FJ, Barbul A. Healing in the gastrointestinal tract. In: Barbul A. Wound healing. Surg Clin North Am. 1997; 77: 547-70.

18. Kuzu MA, Koskoy C, Kale T, Demiprence E, Renda N. Experimental study of the effect of preoperative 5fluorouracil on the integrity of colonic anastomoses. $\mathrm{Br}$ J Surg. 1998;85: 236-9.

19. Koruda MJ, Rolandelli RH. Experimental studies on the healing of colonic anastomoses. J Surg Res. 1990;48: 504-15.

20. Ikeuchi D, Onodera H, Aung T, Kan S, Kawamoto H, Iamamura M, Maetani S. Correlation of tensile strength with bursting pressure in the evaluation of intestinal anastomoses. Dig Surg. 1999; 16: 478-85.

\section{Correspondência}

Antonio Gonçalves Dias

Av. dos Holandeses, qd 29, apt. 302

Ponta D'areia - São Luís -MA

CEP: $65.075-650$

Tel: (98) 3235-6440

antoniouro@oi.com.br
Conflito de interesse: nenhum Fonte de financiamento: Capes

Recebimento: $13 / 01 / 2005$

Revisão: 20/06/2005

Aprovação: 10/04/2006

\section{Como citar este artigo}

Gomes CS, Campos ACL, Torres OJM, Vasconcelos PRL, Moreira ATR, Tenório SB, Tâmbara EM, Sakata K, Moraes Jr H, Ferrer ALS. Efeito do extrato de Passiflora Edulis na cicatrização da parede abdominal de ratos: estudo morfológico e tensiométrico. Acta Cir Bras. [periódico na Internet] 2006;21 Suppl 2:9-16. Disponível em URL: http://www.scielo.

$\underline{\mathrm{br} / \mathrm{acb}}$ 